\title{
Neurophysiological correlates of residual inhibition in tinnitus: Hints for trait-like EEG power spectra
}

\author{
S. Schoisswohl ${ }^{\text {a,* }}$, M. Schecklmann ${ }^{a}$, B. Langguth ${ }^{a}$, W. Schlee ${ }^{\text {a }}$, P. Neff ${ }^{\text {a,b,c }}$ \\ ${ }^{a}$ Department of Psychiatry and Psychotherapy, University of Regensburg, Regensburg, Germany \\ b University Research Priority Program 'Dynamics of Healthy Aging', University of Zurich, Zurich, Switzerland \\ ${ }^{\mathrm{c}}$ Center for Cognitive Neuroscience, University of Salzburg, Austria
}

\section{A R T I C L E I N F O}

\section{Article history:}

Accepted 26 March 2021

Available online 22 April 2021

\section{Keywords:}

Tinnitus suppression

Resting state

Electroencephalography

Acoustic stimulation

Residual inhibition

\section{H I G H L I G H T S}

- Trait-specific forms of oscillatory signatures related to residual inhibition.

- Alpha activity in auditory areas increased during residual inhibition.

- Lack of behavioral and neural correlations hamper conclusive interpretations

\begin{abstract}
A B S T R A C T
Objective: To investigate oscillatory brain activity changes following acoustic stimulation in tinnitus and whether these changes are associated with behavioral measures of tinnitus loudness. Moreover, differences in ongoing brain activity between individuals with and without residual inhibition (RI) are examined (responders vs. non-responders).

Methods: Three different types of noise stimuli were administered for acoustic stimulation in 45 tinnitus patients. Subjects resting state brain activity was recorded before and after stimulation via EEG alongside with subjective measurements of tinnitus loudness.

Results: Delta, theta and gamma band power increased, whereas alpha and beta power decreased from pre to post stimulation. Acoustic stimulation responders exhibited reduced gamma and a trend for enhanced alpha activity with the latter localized in the right inferior temporal gyrus. Post stimulation, individuals experiencing RI showed higher theta, alpha and beta power with a peak power difference in the alpha band localized in the right superior temporal gyrus. Neither correlations with behavioral tinnitus measures nor stimulus-specific changes in EEG activity were present.

Conclusions: Our observations might be indicative of trait-specific forms of oscillatory signatures in different subsets of the tinnitus population related to acoustic tinnitus suppression.

Significance: Results and insights are not only useful to understand basic neural mechanisms behind RI but are also valuable for general neural models of tinnitus.

(c) 2021 International Federation of Clinical Neurophysiology. Published by Elsevier B.V. This is an open access article under the CC BY-NC-ND license (http://creativecommons.org/licenses/by-nc-nd/4.0/).
\end{abstract}

\section{Introduction}

Subjective tinnitus is defined as the perception of a ringing or hissing without the presence of a corresponding internal or external source of sound. If this phantom sound perception is present over a period of at least six months, it is considered as chronic (Mazurek et al., 2010). About 10-15\% of the global population suffers from tinnitus, whereas in $1-2 \%$ it represents a severe burden (Langguth et al., 2013; Heller, 2003; Erlandsson and Dauman,

\footnotetext{
* Corresponding author: Department of Psychiatry and Psychotherapy, University of Regensburg, Universitaetsstrasse 84, 93053 Regensburg, Germany.

E-mail address: stefanschoisswohl@yahoo.de (S. Schoisswohl).
}

2013) with comorbidities such as depression, anxiety disorder, sleep disorder or reduced quality of life (Croenlein et al., 2016; Nondahl et al., 2007; Weidt et al., 2016; Trevis et al., 2016).

Currently there is no treatment option for tinnitus available. A major challenge towards an identification of a treatment is related to heterogeneity in tinnitus phenotypes (Hesse, 2016; Kleinjung and Langguth, 2020; Cederroth et al., 2019; Zenner et al., 2017). Up to now, cognitive behavioral therapy represents the treatment option with the best available evidence for tinnitus (Landry et al., 2020; Cima et al., 2012; Li et al., 2019; Fuller et al., 2020).

In the majority of cases, tinnitus develops as a consequence of cochlear damages subsequent to noise trauma or hearing loss (HL) (Langguth et al., 2013). Typically, the perceived tinnitus pitch 
corresponds to the frequency range of maximum HL (Basile et al., 2013; Roberts et al., 2008; Norena et al., 2002; Schecklmann et al., 2012). Theories about the generation of tinnitus commonly suggest that the reduced or missing auditory input triggers maladaptive alterations along the auditory pathway and the central auditory system, which may lead to the sensation of a phantom sound in the frequencies of the peripheral HL (Eggermont, 2007; Eggermont and Roberts, 2012; Eggermont and Tass, 2015; Adjamian et al., 2009).

On a macroscopic level tinnitus was associated with anomalous oscillatory brain activity patterns such as enhanced activity in the delta and gamma frequency range alongside with reduced alpha activity over temporal regions (Weisz et al., 2005; Weisz et al., 2007). As observed in several neurophysiological investigations, this delta increase and alpha decrease appears to be closely linked to tinnitus perception as well as tinnitus distress (Weisz et al., 2005; Schlee et al., 2014; Adjamian et al., 2012; MoazamiGoudarzi et al., 2010; Balkenhol et al., 2013). Due to relations with tinnitus loudness as defined via tinnitus pitch matching (Balkenhol et al., 2013), subjective tinnitus loudness (van der Loo et al., 2009; De Ridder et al., 2015) or tinnitus-specific increased activity in the auditory cortex (Ashton et al., 2007; Vanneste et al., 2011), high gamma activity was proposed to represent the oscillatory signature of tinnitus perception per se (Weisz et al., 2007). These tinnitus-specific spontaneous brain activity patterns were subsumed under the framework of the thalamo-cortical dysrhythmia model (TCD) (Llinás et al., 1999; Llinás et al., 2005; De Ridder et al., 2015), which was further expanded to the "Synchronization-by-Loss-of-Inhibition-Model" (SLIM) (Weisz et al., 2007).

Conversely, some studies neither observed altered delta and alpha activity in tinnitus (Ashton et al., 2007), any power spectra differences compared to healthy controls (Zobay et al., 2015) nor correlations between electrophysiology and psychoacoustic or psychosocial tinnitus measures (Pierzycki et al., 2016). In the same vein, further studies report higher alpha activity in tinnitus (Moazami-Goudarzi et al., 2010), a relationship of enhanced alpha and tinnitus intensity (Meyer et al., 2014) or emphasize the relevance of other frequency bands like beta and theta in neural activity related to tinnitus (Meyer et al., 2014; Moazami-Goudarzi et al., 2010; Balkenhol et al., 2013). Considering these observations, assumptions about abnormal tinnitus-specific respectively tinnitus-related spontaneous brain activity are not so conclusive as presumed initially.

The phenomenon of short-term tinnitus suppression following acoustic stimulation was first studied almost 50 years ago (Feldmann, 1971; Feldmann, 1983). This phenomenon was defined as "residual inhibition" (RI) and can be observed in $60-80 \%$ of tinnitus sufferers, whereby depth and duration of suppression patterns vary among individuals (Roberts et al., 2006; Roberts, 2007; Vernon and Meikle, 2003). Since that time several experiments already examined the impact of various auditory stimulation techniques on RI. These vary from simple white noise (WN) or pure tones, to the application of specific filters or modulation rates, up to the combination of both modulation techniques applied to WN (Henry et al., 2013; Fournier et al., 2018; Roberts et al., 2008; Roberts et al., 2006; Tyler et al., 2014; Neff et al., 2017; Neff et al., 2019; Reavis et al., 2012; Schoisswohl et al., 2019). It has been suggested that stimulation intensity, duration, specific modulations as well as stimuli including the individual tinnitus frequency (ITF) facilitate short-term acoustic tinnitus suppression.

Another approach to reduce subjective tinnitus loudness for a longer period of time is provided via long-term stimulation with notch filtered music (individual tinnitus pitch is removed from the signal), referred to as "tailor-made notched music training" (TMNMT). The supposed underlying physiological effect behind
TMNMT takes place through an inhibition of frequencies within the notch filter called lateral inhibition. By means of long term applications, maladaptive pathological reorganization of the auditory cortex in tinnitus may be reversed (Pantev et al., 2012; Okamoto et al., 2010).

Nevertheless, little is known about the basic neurophysiological processes behind RI (Roberts, 2007). Reduced firing rates of neurons in the central auditory pathway are theorized to play a key role in RI (Galazyuk et al., 2017; Galazyuk et al., 2019), which covers subcortical structures of the auditory system. There is a paucity in experimental studies examining oscillatory brain activity after acoustic stimulation or rather during RI. With the help of neuromagnetic measures in one tinnitus subject Kristeva-Feige et al. (1995) observed an increase in low frequency $(2-8 \mathrm{~Hz})$ spectral power during RI. Contrary to this observation, single-subject intracranial recordings showed a reduction of low frequency (delta: 1-4 Hz; theta: 4-8 Hz) activity in the auditory cortex during RI (Sedley et al., 2015). These tinnitus-related low frequency oscillations also interacted with alpha $(8-12 \mathrm{~Hz})$, beta $(20-28 \mathrm{~Hz})$ and gamma (>30 Hz) activity (Sedley et al., 2015). Beyond that, tinnitus intensity during RI was identified to be connected to delta (1.5$4 \mathrm{~Hz})$, theta $(4-8 \mathrm{~Hz})$ and gamma $(30-150 \mathrm{~Hz})$ oscillatory activity in the auditory cortex by the use of single patient measurements of neuromagnetic brain activity (Sedley et al., 2012). The relevance of auditory gamma band activity for RI respectively tinnitus perception could be further corroborated by means of an inverse correlation with tinnitus intensity exclusively in tinnitus subjects experiencing residual excitation (Sedley et al., 2012). Kahlbrock and Weisz (2008) evaluated neuromagnetic activity in 10 tinnitus patients experiencing RI, defined as $50 \%$ of tinnitus loudness reduction for $30 \mathrm{~s}$ after stimulation offset. A reduction of delta $(1.3-4 \mathrm{~Hz})$ activity in temporal areas was observed during RI, whereas the gamma band (low: 30.5-49 Hz; high: 50.3-70.2 Hz) was not affected. The authors conclude that during a short-term reduction of tinnitus intensity, tinnitus-related abnormal oscillatory activities are temporary reversed resulting in a restored balance of neural inhibitory and excitatory processes. A recent study from King et al. (2021) investigated ongoing electrophysiological brain activity of 30 tinnitus subjects following broad band noise stimulation. 17 participants were able to experience RI, whereby a comparison of RI with a control auditory stimulation condition without the ability to induce RI revealed differences with respect to ongoing brain activity. In detail, the authors report higher power in the alpha and gamma frequency bands over the course of RI compared to the control condition.

To the best of our knowledge, the above mentioned five studies (Kristeva-Feige et al., 1995; Sedley et al., 2015; Sedley et al., 2012; Kahlbrock and Weisz, 2008; King et al., 2021) represent the only attempts to investigate resting state oscillatory brain activity in the context of RI. The fact that available findings are inconsistent and that merely two experiments - one utilizing Magnetoencephalography (MEG) and one Electroencephalography (EEG) analyzed spontaneous brain activity during RI on a group level indicates an urgent need for respective research whether it is by means of MEG or EEG. Besides single subject analysis, group level analysis represent a basic pillar in science in order to make more general statements about the investigated population e.g., ongoing brain activity associated with RI.

Previous research utilizing neurophysiological measurements, used only one type of non-personalized sound and did not compare participants with and without RI. In the course of this study we are employing an extended set of modified and personalized noise stimuli targeting putatively differential neural mechanisms (i.e., RI and lateral inhibition). Thus the main purpose of this EEG experiment was to examine oscillatory brain activity changes during RI (pre vs. post) following a stimulation with different types of noise. 
Moreover we aimed to investigate, whether these changes are related to subjective tinnitus loudness ratings. Since RI is a phenomenon which cannot be induced in all people with tinnitus, differences in spontaneous brain activity between people who reported RI and those who didn't were analyzed (responders vs. non-responders).

Apart from the efficacy of each used stimulus type in short-term tinnitus suppression on a group level, we hypothesize that filtered noise would result in stronger suppression patterns compared to unfiltered noise. In detail, bandstop-filtered noise is assumed to produce the strongest effect via a potentially suppression of neurons reacting to frequencies within the filter range as already shown in long-term applications via TMNMT (Pantev et al., 2012; Okamoto et al., 2010).

Due to the lack of past research in this field, we have no direct stimulus-specific a priori hypothesis about the types of changes from pre to post auditory stimulation in ongoing brain activity. However, we assume that potential changes in spontaneous brain activity can be associated with subjective tinnitus loudness ratings after stimulation. In accordance to Kahlbrock and Weisz (2008) we expect a decrease in delta and gamma activity as well as an increase in alpha activity from pre to post auditory stimulation in tinnitus cases experiencing RI (responders). Further we anticipate spectral power differences in the respective frequency bands between acoustic stimulation responders and non-responders. In order to link these differences to auditory cortical activation, source localization of the EEG data was performed.

\section{Methods}

\subsection{Participants}

In the course of this study, $\mathrm{N}=45$ (14 female) patients with chronic subjective tinnitus ( $>6$ months tinnitus duration) were recruited from the Interdisciplinary Tinnitus Centre Regensburg, Germany. For participation, patients had to fulfill the following primary inclusion criteria: age between 18 and 75 years; absence of other causes for tinnitus e.g., Meniere's disease, otosclerosis or acoustic neurinoma; no infection of the oropharynx; no present somatic, neurological or psychiatric disorder; no intake of psychoactive medication (e.g., antidepressants or anticonvulsant drugs), respectively substance or alcohol abuse at least 12 weeks before the start of the experiment; no hypersensitivity to sound; no tinnitus frequency $<1 \mathrm{kHz}$; no concurrent participation in other tinnitus-related studies or start of any other tinnitus-related treatment in the last three months prior study start.

Ethical clearance with respect to methodological approach and design was sought from the ethics committee of the University of Regensburg, Germany before commencing the experiment (ethical approval number: 17-819-101). For a detailed descriptive overview and clinical characteristics of the sample see Table 1 . All participants received detailed information about objective, methods, duration and potential side effects of the study. Every participant gave written informed consent before the start of the study and received an appropriate expense allowance after completion of the experiment.

\subsection{Psychometry}

Prior to the start of the experiment, participants were requested to answer a set of questionnaires compiled of German versions of the Tinnitus Handicap Inventory (THI) (Newman et al., 1994; Kleinjung et al., 2007), the Tinnitus Questionnaire (TQ) (Goebel and Hiller, 1994; Hallam et al., 1988), the Tinnitus Sample Case History Questionnaire (TSCHQ) (Langguth et al., 2007), visual ana- log scales (VAS, \%) for tinnitus awareness, loudness and bothersome, as well as the Questionnaire on Hypersensitivity to Sound (GUF) (Blaesing et al., 2010) (participants with a score of $>23$, which constitutes a very severe impairment, were excluded from our analysis). The survey was performed with SoSci Survey (Leiner, 2016).

\subsection{Audiometry}

Participants hearing thresholds were examined with the toolbox MultiThreshold (University of Essex, United Kingdom) using the implemented paradigm absolute threshold (absThreshold) in Matlab (Matlab R2017a; Mathworks, USA). This paradigm is an implementation of the two-alternatives forced-choice threshold estimation algorithm by Green (1993). Sine tones (0.5 s) were used to test participants hearing level for frequencies from 250 up to $8000 \mathrm{~Hz}$ on an octave scale for each ear separately. Starting loudness level was $30 \mathrm{~dB}$ SPL, which was increased by $10 \mathrm{~dB}$ steps until the participants were able to perceive the sound. The loudness level was raised by $2 \mathrm{~dB}$ steps between trials. ER-2 Insert Earphones (Etymotic Research Inc., USA) together with an external soundcard (RME Fireface UCX; Audio AG, Germany) were used for hearing assessment, subsequent matching of the ITF, definition of the sensation level (SL), minimum masking level (MML) (compare Section 2.4) as well as the proper auditory stimulation.

\subsection{Tinnitometry}

Individual tinnitus pitch matching was carried out using a Method of Adjustment approach modified from Henry et al. (2013) and Roberts et al. (2008) and implemented in a custom software tool (MAX 7; Cycling'74, USA). A custom-built hardware controller was used comprising a Teensy 3.2 USB-based microcontroller (PJRC, USA) and industrial-grade rotating knobs, switches and motor faders. Detailed information about the used tinnitus matching procedure is described in Neff et al. (2019). The starting frequency was defined as one frequency group below the frequency with the highest $\mathrm{HL}$ and a start loudness of $10 \mathrm{~dB}$ above the particular hearing threshold. Participants tried to match their tinnitus four times as good as possible and rated the accordance of the matched sound with their perceived tinnitus on a $1-10$ scale ( 1 = no accordance; $10=$ perfect accordance) after each attempt. The tinnitus matching trial with the highest rating was subsequently defined as the participants ITF. If participants rated different matching attempts similarly, the frequency closest to the mean frequency of the four attempts was chosen. The ITF was then used for the evaluation of further audiometric parameters. Similarly, the MML was defined by increasing the loudness of $\mathrm{WN}$ to the point of complete tinnitus masking. Assessment of the loudness discomfort level (LDL) of participants ITF was executed with the discomfort paradigm of the MultiThreshold toolbox with Sennheiser HDA 2000 headphones (Sennheiser, Germany).

\subsection{Acoustic stimulation}

Three different types of noise stimuli with a duration of three minutes each were created in Matlab (Matlab R2017a; Mathworks, USA) with an intensity of $65 \mathrm{~dB}$ SL (defined as the loudness level of participants first-time tinnitus pitch perception; maximum loudness of $85 \mathrm{~dB}$ SPL) for acoustic stimulation. For this purpose a genuine $\mathrm{WN}$ was used to produce individualized noise stimuli through the implementation of bandpass (IBP) and bandstop (IBS) filters with one octave width around the ITF (Pantev et al., 2012). Each stimuli was composed of a $1000 \mathrm{~ms}$ linear fade-in and fade-out phase and underwent a root-mean-square correction to balance levels between stimuli. Diotic acoustic stimulation was performed 
Table 1

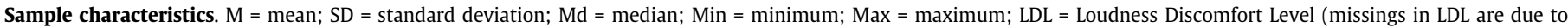
values over $90 \mathrm{~dB}$ ); TQ = Tinnitus Questionnaire; THI = Tinnitus Handicap Inventory; VAS = Visual Analog Scale; GUF = Questionnaire on Hypersensitivity to Sound.

\begin{tabular}{|c|c|c|c|c|}
\hline $\begin{array}{l}\mathrm{N} \text { (female) } \\
\text { Tinnitus side (left/ right/ bilateral) } \\
\text { Tinnitus loudness fluctuation (yes/ no) } \\
\text { Tinnitus maskability (yes/ no/ don't know) } \\
\text { Musician (yes/ no) }\end{array}$ & $\begin{array}{l}45(14) \\
(5 / 8 / 32) \\
(24 / 21) \\
(31 / 5 / 9) \\
(4 / 41) \\
M \pm \text { SD }\end{array}$ & Md & Min & Max \\
\hline Age (years) & $52.29 \pm 11.81$ & 55.00 & 23.00 & 69.00 \\
\hline Tinnitus duration (months) & $111.04 \pm 72.90$ & 96.00 & 18.00 & 280.00 \\
\hline Tinnitus frequency $(\mathrm{Hz})$ & $6251.09 \pm 2811.38$ & 5887.00 & 1020.00 & 15524.00 \\
\hline Tinnitus loudness (dB SPL) & $51.38 \pm 16.05$ & 50.00 & 27.00 & 85.00 \\
\hline Hearing loss left $(\mathrm{dB})$ & $17.26 \pm 13.61$ & 14.69 & -5.72 & 55.00 \\
\hline Hearing loss right $(\mathrm{dB})$ & $17.48 \pm 11.52$ & 17.43 & -8.71 & 45.87 \\
\hline LDL left (dB) (25 missing values) & $86.25 \pm 3.21$ & 85.50 & 81.00 & 90.00 \\
\hline LDL right (dB) (28 missing values) & $85.06 \pm 3.96$ & 87.00 & 78.00 & 90.00 \\
\hline Minimum masking level $(\mathrm{dB})$ & $63.82 \pm 14.60$ & 60.00 & 37.00 & 90.00 \\
\hline Sensation Level $(\mathrm{dB})$ & $47.58 \pm 17.49$ & 45.00 & 21.00 & 86.00 \\
\hline TQ total score $(0-84)$ & $40.73 \pm 15.70$ & 40.00 & 17.00 & 71.00 \\
\hline THI total score $(0-100)$ & $35.91 \pm 21.38$ & 34.00 & 4.00 & 80.00 \\
\hline VAS awareness (\%) & $64.62 \pm 29.62$ & 70.00 & 8.00 & 100.00 \\
\hline VAS loudness (\%) & $61.11 \pm 24.19$ & 65.00 & 15.00 & 100.00 \\
\hline VAS bothersome (\%) & $38.20 \pm 29.29$ & 30.00 & 0 & 100.00 \\
\hline GUF total score $(0-45)$ & $10.73 \pm 6.45$ & 10.00 & 0 & 23.00 \\
\hline
\end{tabular}

at a maximum loudness of $85 \mathrm{~dB}$ SPL and each stimuli was presented only once. The presentation sequence of the stimuli was randomized.

Before and after the presentation of each stimuli ( $3 \mathrm{~min}$ ), participants were requested to sit quietly, focus on a white fixation cross on a black screen and avoid extensive eye-blinks and movements while their brain activity was recorded via EEG for three minutes respectively (compare Section 2.7).

After the presentation of each noise stimulus, patients had to rate the loudness of their tinnitus at seven different time points (0sec, 30sec, 60sec, 90sec, $120 \mathrm{sec}, 150 \mathrm{sec}$ and $180 \mathrm{sec}$ after stimulation offset) on a customized keyboard strip (X-Key-Stick-16-USB, XK-0981-UCK16-R; P.I. Engineering, USA) with a numeric rating scale from $0 \%$ to $110 \%$, whereas $100 \%$ signified no tinnitus loudness changes, $0 \%$ a total absence of tinnitus and $110 \%$ an tinnitus loudness increase by $10 \%$. For an illustration of the acoustic stimulation procedure please see Fig. 1. The whole experimental stimulation procedure was implemented with the Psychophysics Toolbox Version 3 (Brainard, 1997; Kleiner et al., 2007) in Matlab (Matlab R2017a; Mathworks, USA) and double-blinded. At the end of the experiment, the three stimuli were again presented in a randomized order for $10 \mathrm{~s}$ each and participants were requested to rate the valence and the arousal of each stimuli via pictorial manikin scales (Bradley and Lang, 1994) on a 9-point Likert Scale, whereas the value 0 indicated a neutral stimulus evaluation (Valence: -4 unpleasant, 4 pleasant; Arousal: -4 relaxing, 4 upsetting).

\subsection{Behavioral analysis}

Behavioral data was analyzed with the statistic software $R$ ( $R$ version 3.4.2; $\mathrm{R}$ Foundation for Statistical Computing, Austria) and the packages "psych", "emmeans", "sjstats" and "lme4". Linear mixed effect models were used to analyze tinnitus loudness ratings and stimuli evaluation (valence, arousal) separately. The following predictors were tested for the model fitting procedure of tinnitus loudness ratings: condition (stimuli, compare Section 2.5), time (0sec, 30sec, 60sec, 90sec, 120sec, 150sec, 180sec towards stimulation offset), tinnitus bilaterality (yes/no), sex (male/female), tinnitus duration and stimuli position in the auditory stimulation sequence. The predictors condition, gender and tinnitus duration were tested for the model fitting procedure of stimuli evaluation data.

Other potential predictors such as tinnitus loudness (dB), MML, SL or HL were not included in the model fitting procedure, since they were experimentally controlled e.g., by the creation of tailored stimuli. Participant (id) was considered as a random effect in all model fitting procedures. In order to identify the model with the best fit for the data, the step function of the lme4 package was deployed. Thereby, a backward elimination of non significant predictors as well as a forward addition of significant predictors is conducted by comparing the models with Likelihood Ratio Tests (Harrison et al., 2018). Marginal (variance of the predictors) and conditional (variance of predictor and random effect) $R^{2}$ were

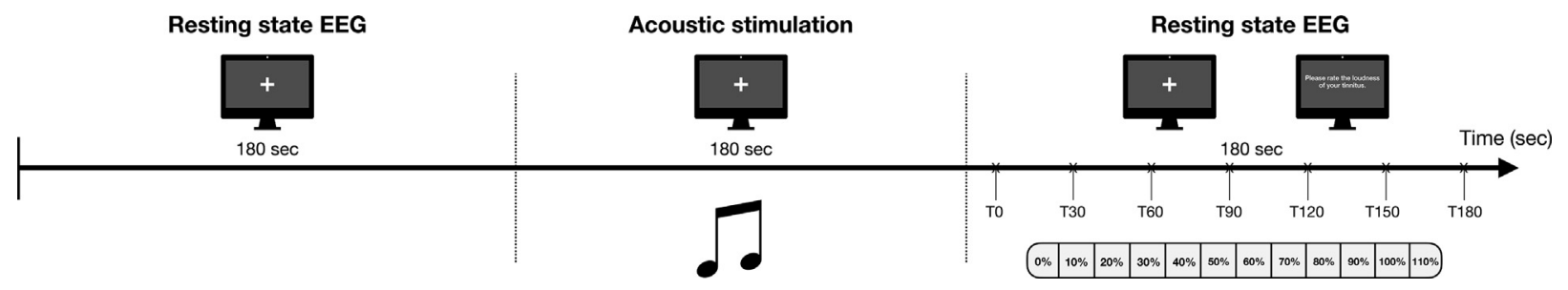

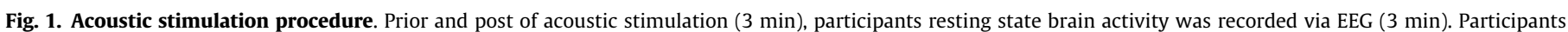

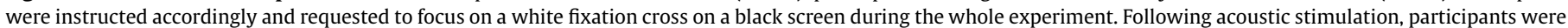

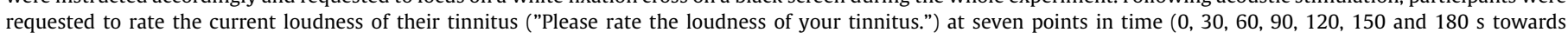

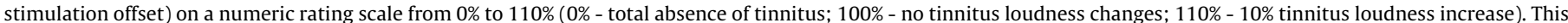

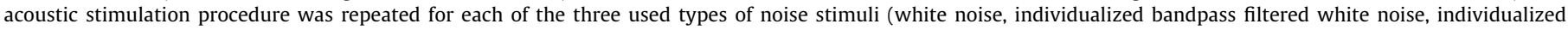
bandstop filtered white noise). 
computed to provide the amount of the explained variance of the respective model (Nakagawa et al., 2017). For each final model, fixed effects were examined via Expected Mean Square Approach. Potential differences in tinnitus loudness and stimuli evaluation within predictors were analyzed with post hoc Tukey-tests. Analysis of descriptive differences between HL and LDL between the left and right ear were tested by the means of two-sample t-tests. Normal distribution (Shapiro-Wilk-Test) and homoscedasticity (Ftest) were examined and if violated, non-parametric testing with independent sample Mann-Whitney U-tests were conducted. To evaluate effect size of significant differences, Cohen's d was calculated. The level of statistical significance was set to $p \leqslant .05$ for all analyses.

\subsection{Electrophysiological data acquisition and analysis}

\subsubsection{EEG recording}

EEG data was recorded with a BrainAmp DC system, EasyCap electrode cap with 64 electrodes, and Brain Vision Recorder 1.20 software (Brain Products $\mathrm{GmbH}$, Germany). The sampling rate was $500 \mathrm{~Hz}$ and electrodes were referenced to $\mathrm{FCz}$ during recording. Impedances were kept below $10 \mathrm{k} \Omega$.

\subsubsection{Preprocessing}

Raw EEG data was preprocessed with a custom-built semiautomatic pipeline using the Fieldtrip toolbox (Oostenveld et al., 2011) in Matlab (Matlab R2017a; Mathworks, USA). EEG data was filtered between $0.5 \mathrm{~Hz}$ and $45 \mathrm{~Hz}$ with a 4th order Butterworth bandpass filter.

Hereafter, an independent component analysis (ICA, fastICA http://research.ics.aalto.fi/ica/fastica/index.shtml) was used to identify and remove components with horizontal and vertical eye movement. Noisy or aberrant channels were interpolated using weighted neighbors. Neighboring channels were defined via a triangulation of 2D sensor position projection and channels identified for interpolation were replaced with the mean of neighboring sensors. In a next step, average referencing was performed and the recording reference electrode $\mathrm{FCz}$ was added as a data channel. In order to control for noisy channels introduced by the rating procedure of the post stimulation conditions, posterior (Iz, TP9, TP10) as well as frontal channels (FPz, FP1, FP2, AF3, AF4, AF7, AF8) were discarded from subsequent analyses steps. Data was then segmented into $2 \mathrm{~s}$ segments. All segments during which participants rated the loudness of their tinnitus were rejected. Additionally, one segment before and after the rating was excluded as well. Segments with remaining artifacts were rejected with combined automatic identification via a $z$-score $(\mu \mathrm{V})$ threshold of $-2 /+2$ and visual inspection in a final step. Average number of valid segments was different $(U=1970.50, p=.001)$ between pre $(M=78.93$, $\mathrm{SD}=6.48)$ and post $(\mathrm{M}=60.37, \mathrm{SD}=6.19)$ acoustic stimulation.

\subsubsection{EEG analysis}

Power analysis - whole group Frequency power spectra of pre and post auditory stimulation datasets per subject and condition (compare 2.5) were calculated using multitaper frequency transformation (mtmfft) and a hanning window with a spectral smoothing of $1 \mathrm{~Hz}$. Next, grand averages were created for pre and post stimulation datasets per condition by computing power spectra averages across all valid segments and all subjects.

Potential changes in EEG power spectra were analyzed with a 2 $\mathrm{x} 3$ repeated measurement ANOVA and the within subject factors time (pre, post) and condition (WN, IBP, IBS), which was implemented in Fieldtrip. The main effects for time and condition were tested with paired two-sided t-tests via non-parametric clusterbased permutation tests with 10.000 iterations. In order to test for an interaction effect of time and condition, a dependent sam- ples multivariate ANOVA was conducted using a non-parametric cluster-based permutation test with 10.000 iterations as well. We were primary interested in an interaction effect of time and condition. In case of a significant time $\mathrm{x}$ condition interaction, effects were followed up using post hoc contrasts. Pre vs. post contrast per condition were analyzed with dependent samples t-tests, whereas potential differences in stimuli-induced power spectra changes from pre to post stimulation as well as post stimulation differences (inter-stimulus contrasts), were contrasted via independent samples t-tests using non-parametric cluster-based permutation test as described above.

Additionally, Pearson correlations between post stimulation power spectra and pre-post power spectra differences with averaged tinnitus loudness ratings (over all 7 time points) as well as directly after stimulation offset (T0) were computed via clusterbased permutation tests. Significance level was set to $p \leqslant .05$ for all EEG analyses and $\mathrm{p}<0.1$ was defined as a statistical trend. Significant clusters were defined as a minimum of two significant neighboring channels for all analysis. For the purpose of interpretation, EEG frequency bands were defined as follows: delta 1$4 \mathrm{~Hz}$, theta $5-7 \mathrm{~Hz}$, alpha $8-12 \mathrm{~Hz}$, beta $13-29 \mathrm{~Hz}$, gamma $30-$ $45 \mathrm{~Hz}$.

Power analysis - responder Furthermore, we compared frequency power spectra of participants who exhibited RI with those who did not experience RI after auditory stimulation. For this purpose RI was defined as $\leqslant 50 \%$ of tinnitus loudness directly after stimulation offset resulting in a subset of $n=12$ further indicated as responders. Within this subgroup of responders, $n=5$ participants each, responded to a stimulation with WN or IBP, whereas only $\mathrm{n}=2$ participants reported RI after a stimulation with IBS. A second subgroup of participants without RI (non-responders) were matched to responders according to the following criteria: gender; mean HL; age and absence of RI (tinnitus loudness of $\geqslant 100 \%$ after stimulation offset) in the same stimulus type as matched patient exhibited RI in responders group. Sample characteristics for both subgroups can be seen from Table 2. Associations of categorical variables with stimulation response (responder or nonresponder) were analyzed with $\chi^{2}$-tests or Fisher's exact tests if cell frequencies were below 5. Differences in numerical variables between the two subgroups were analyzed by two-sample ttests. In case of violated statistical assumptions, Mann-Whitney U-tests were performed. Significance levels were set to $p \leqslant .05$ and a statistical trend was defined as $\mathrm{p}<0.1$.

Power spectra for pre and post auditory stimulation EEG datasets were averaged over all subjects within the respective subgroup (responders and non-responders). Analysis were conducted using normalized EEG datasets by dividing power spectra for each single frequency through the total power of the entire frequency spectrum according to the formula:

$W(f)_{\text {norm }}=\frac{W(f)}{\int_{f_{-} 1}^{f_{-} 45} W(f) d f}$

Illustrated power spectra per frequency were transformed according to $10^{*} \log 10(\mathrm{x})$. EEG power spectra were analyzed with a $2 \mathrm{x}$ 2 repeated measures ANOVA and the factors time (pre, post) and group (responders, non-responders). The main effects for time and group were evaluated with dependent sample respectively independent sample t-tests according to the same approach as already described in the power analysis section for the whole group. Likewise, a potential interaction effect of time and group was analyzed with an independent samples t-test.

In the case of a significant interaction effect, post hoc dependent samples t-tests for pre vs. post within subgroup contrast and independent samples t-tests for between subgroup contrast (responders vs. non-responders) separated for pre and post stimulation 
measurements are conducted. Regardless of an observed interaction effect, an exploratory contrast of post stimulation power spectra differences between responders and non-responders is performed. Equal to the whole group analysis, Pearson correlations were calculated with cluster-based permutation tests for post stimulation power spectra and pre-post power spectra differences with averaged tinnitus loudness ratings or rather directly after stimulation offset (T0). Additionally, a correlation of post stimulation power spectra and pre-post power spectra differences with tinnitus loudness rated via VAS (\%) was computed.

In order to explore differences in cortical alpha variability between responders and non-responders a coefficient of variance was calculated by dividing the standard deviation of the alpha frequency power $(8-12 \mathrm{~Hz})$ by its mean power.

Source space analysis Source localization of frequency data was performed using a standard boundary element headmodel (Oostenveld et al., 2003) and the dynamic imaging of coherent sources algorithm optimized for EEG frequency data (Dynamical Imaging of Coherent Sources, (Groß et al., 2001)). First, crossspectral density was calculated for each electrode using ' $m$ tmfft' with the 'powandcsd' option and a hanning window with $1 \mathrm{~Hz}$ spectral smoothing in the peak frequency extracted from the scalp analysis. Second, a standard boundary element headmodel including the tissues of scalp, skull, and brain was used ('standard_bem'). Details of the segmentation and the conductivity models are described in Oostenveld et al. (2003, 2002). An adapted standard electrode layout was used ('standard_1020') where the noisy channels described above were dropped. Electrode alignment was then checked visually and alignment optimized so that the electrodes were correctly positioned over the scalp and not part of any tissue of the headmodel. Finally, the leadfield was calculated with the headmodel and the aligned electrodes. Notably, a single headmodel, electrode, and leadfield template was created for source analysis of all participants given the absence of individual MRIs and electrode positions.

Inter-subgroup source contrasts (responders vs. nonresponders; responders vs. non-responders post stimulation) of peak frequencies derived from the respective sensor-level cluster analysis (maximum value; please see Section 3.5 under the subheading for responder) were analyzed via non-parametric cluster-based permutation tests with 10.000 iterations using normalized EEG datasets. Normalization procedure was identical to the sensor level analysis.

\section{Results}

\subsection{Sample characteristics}

Table 1 summarizes the descriptive statistics and tinnitusrelated questionnaire scores of the present sample. In the majority of participants, tinnitus was perceived bilaterally ( $n=32$ ) and featured loudness fluctuations $(n=24)$. The possibility to mask their perceived tinnitus was reported by $n=31$ participants. Moreover, $\mathrm{n}=4$ participants claimed to be musicians and the average duration of tinnitus perception was 111.04 months (SD = 72.90).

Stimulation with either WN, IBP and IBS resulted in $n=12$ responders, who showed RI with at least one stimulus type.

A weak association of stimulation response (responders or nonresponders) and tinnitus maskability (yes, no, don't know) was found with the group of responders exhibiting no participant who reported an absence of tinnitus maskability (cf. Table 2). Statistical testing for differences between the subgroups of responders and non-responders revealed differences in terms of tinnitus duration, MML and questionnaire data with the group of responders showing shorter tinnitus duration $(U=26.00, p=.008, d=1.135)$,

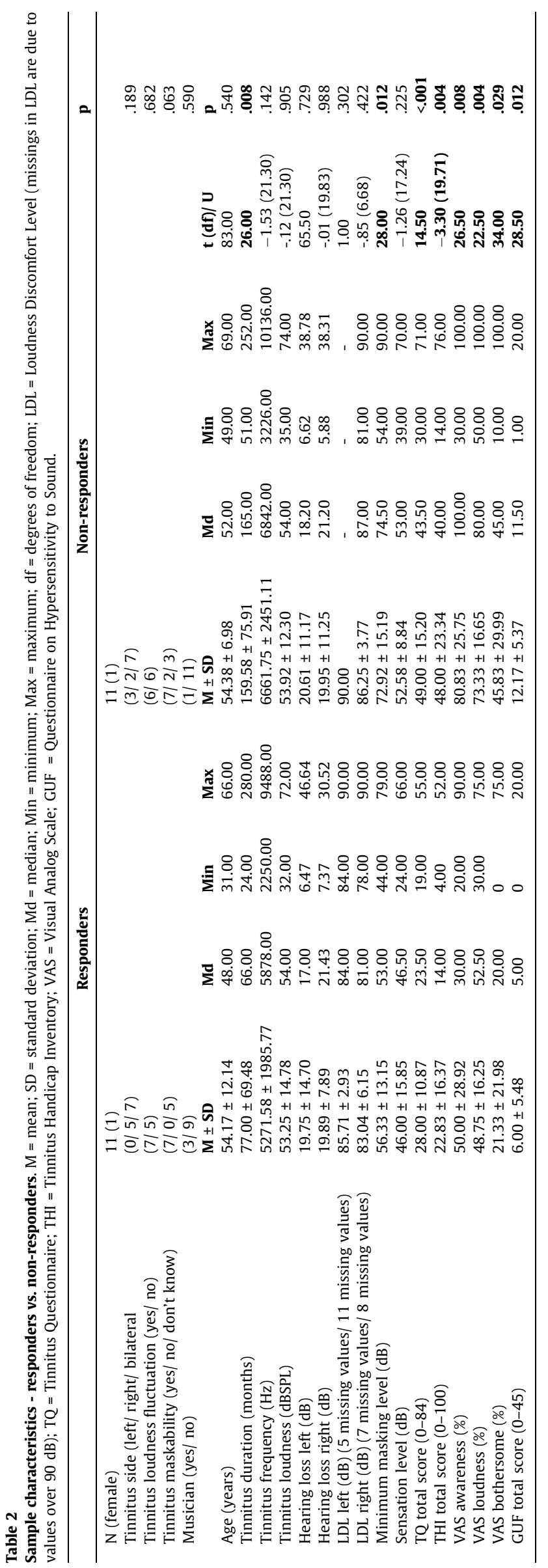


lower MML $(\mathrm{U}=28.00, \mathrm{p}=.012 . \mathrm{d}=1.168)$ as well as lower sum scores in TQ $(U=14.50, \mathrm{p}<.001, \mathrm{~d}=1.159)$, THI $\left(\mathrm{t}_{(19.71)}=-3.30\right.$, $\mathrm{p}=.004, \mathrm{~d}=1.249)$ and GUF $(\mathrm{U}=28.50, \mathrm{p}=.012, \mathrm{~d}=1.137)$. Likewise, responders reported lower values in subjective measurements of tinnitus awareness $(U=26.50, p=.008, d=1.126)$, loudness $(\mathrm{U}=22.50, \mathrm{p}=.004, \mathrm{~d}=1.494)$ and bothersome $(\mathrm{U}=34.00, \mathrm{p}=.029, \mathrm{~d}=.931)$ as indicated by VAS (in \%). Detailed sample characteristics and statistical comparisons for the two subgroups are shown in Table 2.

\subsection{Audiometry and Tinnitometry}

Results from audiometric assessment and tinnitus matching are outlined in Table 1 as well as illustrated in Fig. S1. The investigated sample featured a mean tinnitus frequency of $6251.09 \mathrm{~Hz}$ $(S D=2811.38)$, whereas the average tinnitus loudness was $51.38 \mathrm{~dB}$ SPL $(\mathrm{SD}=16.05)$. Initial perception of the individual tinnitus pitch (SL) appeared at a mean volume level of $47.58 \mathrm{~dB}$ $(\mathrm{SD}=17.49)$. Mann-Whitney U-tests found no differences with respect to $\mathrm{HL}(\mathrm{U}=941.50, \mathrm{p}=.569)$ and $\operatorname{LDL}(\mathrm{U}=199.50, \mathrm{p}=.361)$ between the left and the right ear.

\subsection{Acoustic Stimulation}

Table S1 lists the descriptive statistics for tinnitus loudness ratings for each stimuli on average as well as time point T0. Tinnitus suppression time curves, including all seven time points, are illustrated in Fig. 2 for each stimuli.

Model fitting procedure of behavioural data was able to identify the following model with the best fit for the data: response $\sim$ condition $+(1 \mid i d)$. Table S3 lists detailed results of the model fitting proceeding. A significant effect of condition was observed (cf. Table S3). Suceeding post hoc contrasts found differences between stimulus WN vs. IBS, as well as IBP vs. IBS (cf. Table 3). A potential confounding caused by the position of the stimuli in the acoustic stimulation sequence could be excluded, since position did not appear as a significant predictor in the final model.

\subsection{Stimulus evaluation}

Stimulus evaluation outcomes in terms of valence and arousal can be seen from Table S4 and Fig. S2. Model response $\sim$ condition $+(1 \mid$ id $)$ was identified to have the best fit for the valence data with condition as a significant fixed effect (cf. Tables S5 and S6). Post hoc tests were able to reveal differences for valence evaluations of stimuli WN vs. IBS and also IBP vs. IBS as can be seen from Table S7. Subsequent model was identified by our model fitting approach for arousal data: response $\sim$ condition + gender $+(1 \mid$ id $) \quad($ cf. Table S5). Fixed effect testing revealed significant effects for condition and gender (cf. Table S6). Post hoc analysis showed differences between stimuli IBP and IBS as well as male and female participants (cf. Table S7).

\subsection{Electrophysiology}

Whole group Results of whole sample EEG power spectra analysis are outlined in Table 4. A significant main effect of time was observed, indicating higher spectral power for $1-7 \mathrm{~Hz}$ and 26$45 \mathrm{~Hz}$ plus lower spectral power for 7-28 $\mathrm{Hz}$ after auditory stimulation. Further, a significant interaction of condition and time was found in the frequency spectra 1-7 Hz and 36-45 Hz. Succeeding post hoc contrasts revealed higher power in lower frequencies towards stimulation across all stimuli (WN: 1-7 Hz; IBP: 1-6 Hz;
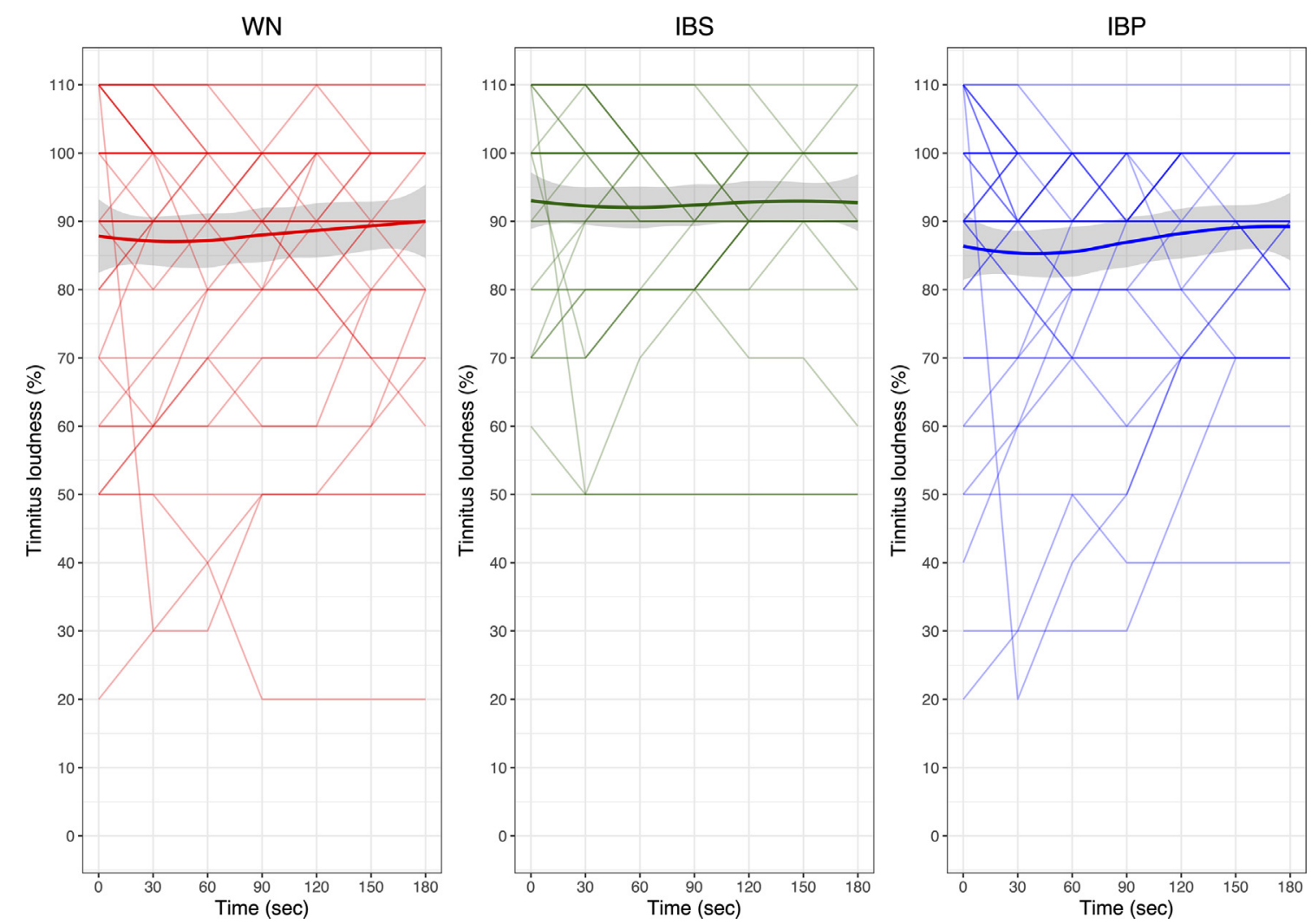

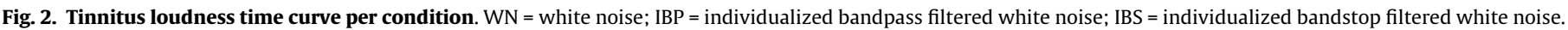

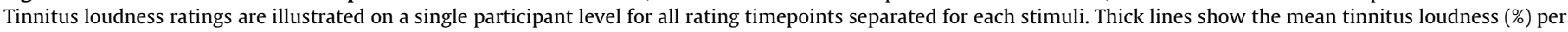
stimulus, standard deviations are illustrated as grey ribbons. 
Table 3

Post hoc tukey contrasts for condition. $\mathrm{WN}=$ white noise; $\mathrm{IBP}=$ individualized bandpass filtered white noise; IBS = individualized bandstop filtered white noise; degrees of freedom $=902.00$; standard error $=.87$.

\begin{tabular}{lllll}
\hline Contrast & Estimate & $\mathbf{t}$ & $\mathbf{p}$ & $\mathbf{d}$ \\
\hline Total sample & & & & \\
WN - IBP & 1.05 & 1.20 & .451 & .057 \\
WN - IBS & -4.32 & $-\mathbf{4 . 9 6}$ & $<.001$ &. $\mathbf{2 5 1}$ \\
IBP - IBS & $\mathbf{5 . 3 7}$ & $-\mathbf{6 . 1 7}$ & $<.001$ & $\mathbf{. 3 2 8}$ \\
\hline
\end{tabular}

IBS: 1-6 Hz) as well as higher gamma activity after a stimulation with IBP (32-45 Hz) and IBS (37-45 Hz). A power decrease following IBS stimulation was found for the frequency cluster 11-19 Hz. In addition, statistical trends towards power reductions in the frequency clusters $10-12 \mathrm{~Hz}$ and $14-19 \mathrm{~Hz}$ were observed for pre-post comparisons of stimulus WN. Differences between the applied types of stimuli with respect to pre-post power spectra changes or post stimulation power spectra were not detected.

Electrodes within frequency clusters as outlined in Table 4 can be found in the supplemental material in Table S8 grouped by brain areas.

No correlations were found on the cluster level for post stimulation EEG power or pre-post power spectra changes with averaged tinnitus loudness ratings or rather tinnitus loudness ratings immediately after stimulation end (T0) for any of the used stimuli.
Responder Table 5 provides the results obtained from the responder EEG power spectra analysis (compare Section 2.7.3). A significant main effect of time was observed, indicating a power reduction from pre to post stimulation in the frequency cluster 6-32 $\mathrm{Hz}$ for responders as well as non-responders. Likewise, a significant effect of group demonstrates lower power in higher frequency ranges $(22-45 \mathrm{~Hz} ; \mathrm{t}(\max )=-4.06$, over electrode $\mathrm{P} 5$ at $31 \mathrm{~Hz}$; cf. Fig. $3 \mathrm{~A}$ and $\mathrm{B}$ ) as well as a statistical trend towards higher power in the alpha frequency range $(7-12 \mathrm{~Hz} ; \mathrm{t}(\max )=$ 4.35, over electrode F4 at $9 \mathrm{~Hz}$; cf. Fig. $3 \mathrm{~A}$ and B) for the subgroup of responders. There was no significant interaction of time and group. Electrodes within frequency cluster presented in Table 5 can be found in Table S9 in the supplemental material.

Subsequent exploratory analysis of post stimulation power spectra differences between responders and non-responders, exhibited increased activity in the frequency cluster $5-17 \mathrm{~Hz}$ in the subgroup of responders $(t(\max )=4.94$, over electrode $\mathrm{F} 4$ at $9 \mathrm{~Hz}$; cf. Table 5 and Fig. $4 \mathrm{~A}$ and B).

Correlations of EEG power post stimulation or pre-post power spectra changes on the cluster level with subjective tinnitus ratings for the group of responders showed no significant results for mean tinnitus loudness or tinnitus loudness at T0. Further no correlation with tinnitus loudness rated via VAS (\%) was observed.

Coefficient of variance calculation exclusively for the alpha frequency band $(8-12 \mathrm{~Hz}$ ) exposed a higher variation in frequency

Table 4

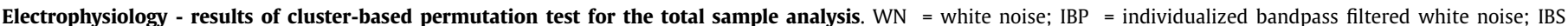

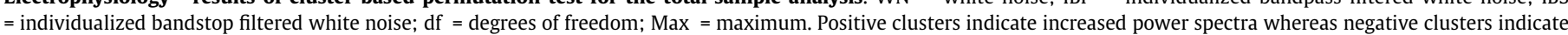

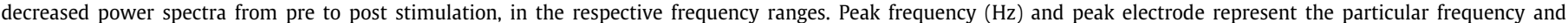
electrode featuring the maximum value obtained from cluster statistics.

\begin{tabular}{|c|c|c|c|c|c|c|}
\hline & Frequency $(\mathrm{Hz})$ & Cluster statistic (df) & $\mathbf{p}$ & Peak frequency $(\mathrm{Hz})$ & Peak electrode & Max. statistic \\
\hline \multicolumn{7}{|l|}{ Time } \\
\hline Positive cluster & $1-7$ & $\mathrm{t}(134)=1047.88$ & $<.001$ & 4 & PO8 & 7.68 \\
\hline Positive cluster & $26-45$ & $t(134)=893.13$ & $<.001$ & 41 & $\mathrm{POz}$ & 4.89 \\
\hline Negative cluster & $7-28$ & $t(134)=-1150.64$ & $<.001$ & 12 & T8 & -5.33 \\
\hline \multicolumn{7}{|l|}{ Condition $\mathrm{x}$ Time } \\
\hline Positive cluster & $1-7$ & $\mathrm{~F}(5,40)=3437.77$ & .002 & 4 & PO8 & 51.28 \\
\hline Positive cluster & $36-45$ & $F(5,40)=2783.52$ & .002 & 42 & F6 & 34.09 \\
\hline \multicolumn{7}{|c|}{$\begin{array}{l}\text { Post hoc - pre vs. post } \\
\text { stimulation per stimulus }\end{array}$} \\
\hline \multicolumn{7}{|c|}{ Positive cluster } \\
\hline WN & $1-7$ & $\mathrm{t}(44)=482.28$ & .006 & 5 & 01 & 4.81 \\
\hline IBP & $1-6$ & $t(44)=696.17$ & .002 & 3 & 01 & 5.90 \\
\hline IBP & $32-45$ & $\mathrm{t}(44)=460.98$ & .007 & 41 & F3 & 4.44 \\
\hline IBS & $1-6$ & $t(44)=398.13$ & .006 & 3 & $\mathrm{O} 2$ & 4.20 \\
\hline IBS & $37-45$ & $\mathrm{t}(44)=199.09$ & .026 & 45 & P2 & 3.54 \\
\hline \multicolumn{7}{|l|}{ Negative cluster } \\
\hline WN & $10-12$ & $t(44)=-132.92$ & .058 & 11 & $\mathrm{~T} 8$ & -4.24 \\
\hline WN & $14-19$ & $t(44)=-123.90$ & .064 & 19 & $\mathrm{C} 3$ & -3.95 \\
\hline IBS & $11-19$ & $\mathrm{t}(44)=-242.31$ & .016 & 13 & T8 & -4.20 \\
\hline
\end{tabular}

Table 5

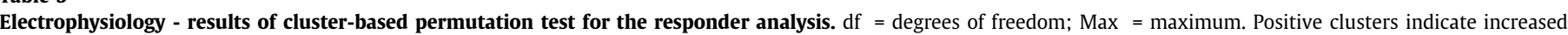

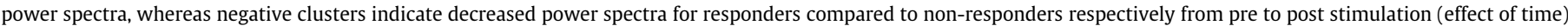

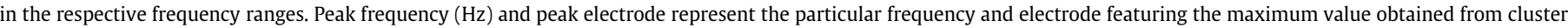
statistics.

\begin{tabular}{|c|c|c|c|c|c|c|}
\hline & Frequency $(\mathrm{Hz})$ & Cluster statistic (df) & $\mathbf{p}$ & Peak frequency $(\mathrm{Hz})$ & Peak electrode & Max. statistic \\
\hline \multicolumn{7}{|l|}{ Time } \\
\hline Negative cluster & $6-32$ & $\mathrm{t}(11)=-1539.00$ & $<.001$ & 18 & TP7 & -6.77 \\
\hline \multicolumn{7}{|l|}{ Group } \\
\hline Positive cluster & $7-12$ & $t(22)=246.27$ & .082 & 9 & $\mathrm{~F} 4$ & 4.35 \\
\hline Negative cluster & $22-45$ & $t(22)=-573.34$ & .024 & 31 & P5 & -4.06 \\
\hline \multicolumn{7}{|c|}{$\begin{array}{l}\text { Exploratory post hoc contrast - responders vs. } \\
\text { non-responders post stimulation }\end{array}$} \\
\hline Positive cluster & $5-17$ & $\mathrm{t}(22)=549.39$ & .035 & 9 & $\mathrm{~F} 4$ & 4.94 \\
\hline
\end{tabular}


A

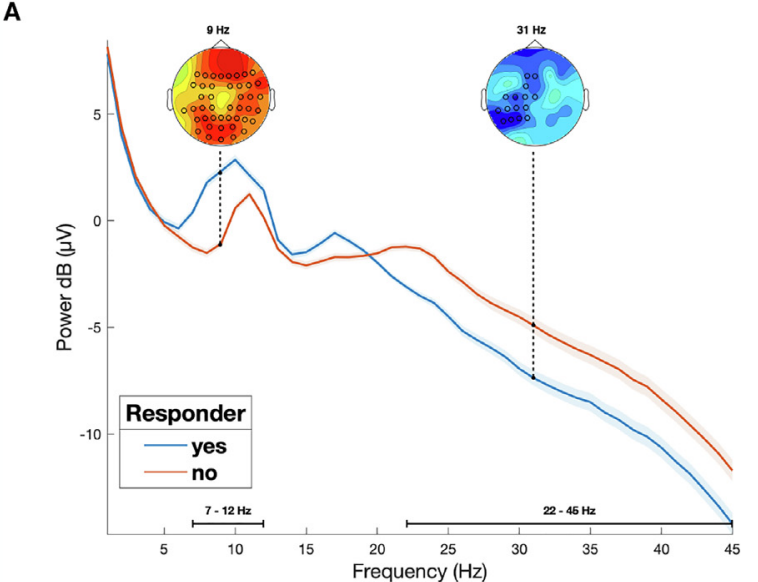

$9 \mathrm{~Hz}$ - right inferior temporal cortex

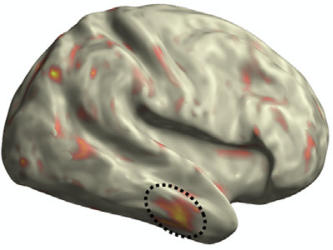

MNI [60 - $10-30]$
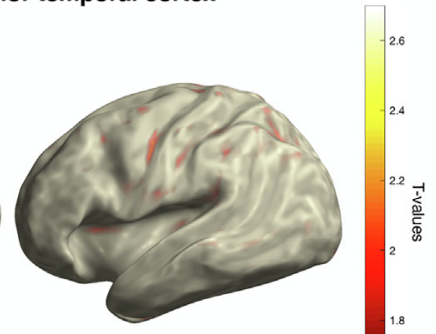

(3)

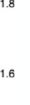

B

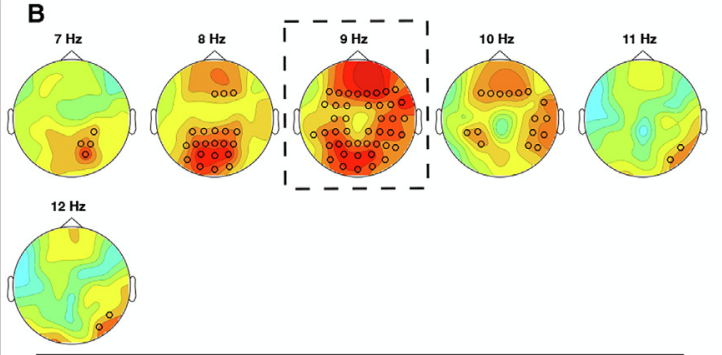

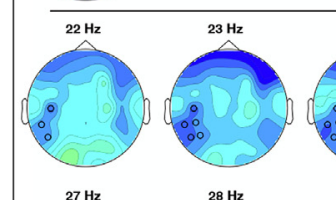
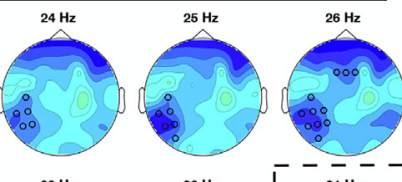

I $-\frac{-}{31 \mathrm{~Hz}}-1$
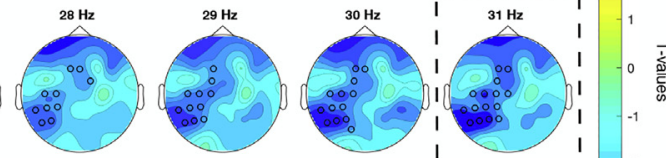
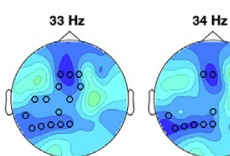

$34 \mathrm{~Hz}$
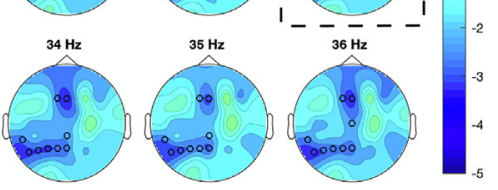

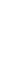
Ф
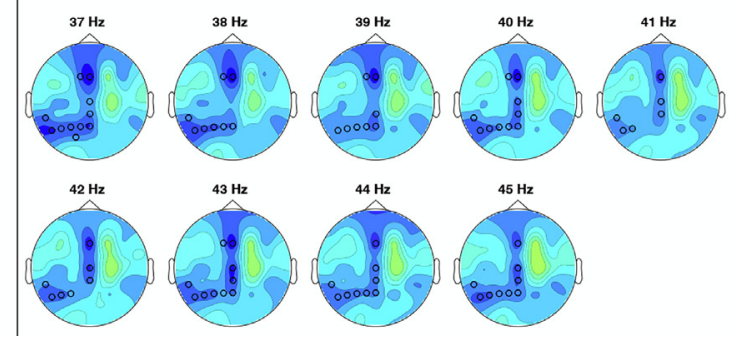

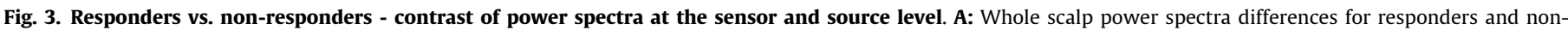

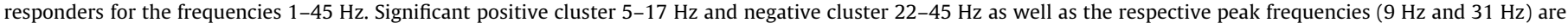

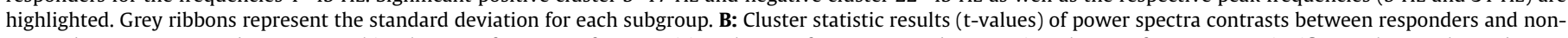

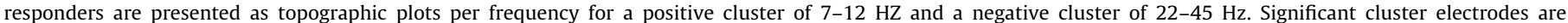

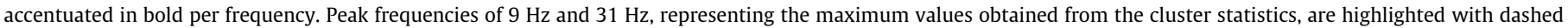
line rectangles. C: Source localization of $9 \mathrm{~Hz}$ EEG power peaking in the right inferior temporal gyrus (BA 20).

band power for the subgroup of responders (responders: 61.04\%; non-responders: $50.03 \%$ )

Source localization Projecting peak frequencies of sensor-level power differences of responders and non-responders contrasts in source space exposed differences solely for $9 \mathrm{~Hz}$ ( $\mathrm{t}$ (cluster) $=$ 13.07, $\mathrm{p}=.004)$ with maximum differences $(\mathrm{t}(\max )=2.70)$ localized in the right inferior temporal gyrus (MNI: $60-10-30$ ) shown in Fig. 3C). However, no difference at the peak frequency $31 \mathrm{~Hz}$ could be observed in source space. Source localization of the peak frequency received from sensor-level contrast between responders and non-responders post acoustic stimulation exhibited differences at the frequency of $9 \mathrm{~Hz}(\mathrm{t}$ (cluster) $=31.95, \mathrm{p}=.032$ ) localized in the right superior temporal gyrus (MNI: $40-3010$ ) presented in Fig. 4C.

\section{Discussion}

The main objective of the present study was to investigate the effect of different types of noise stimuli on short-term tinnitus suppression and corresponding electrophysiological brain activity. Moreover, we wanted to elucidate if electrophysiological changes are a function of tinnitus loudness ratings and if differential activation patterns arise from the different stimuli putatively triggering RI or lateral inhibition, respectively. Finally, we aimed at examin- ing potential differences in ongoing brain activity between responders and non-responders. To the best of our knowledge, this presentation of notch- and bandpass-filtered WN sounds is novel in its application in tinnitus research. Similarly, we are the first group which elucidated neurophysiological differences between acoustic stimulation responders and non-responders. In the following, the results of our study are thus critically discussed in the light of current knowledge and with respect to future research outlook.

\subsection{Behavioral results}

The behavioral analysis demonstrate similar suppression patterns as past studies in this field with only a subset of the study population reporting a considerable tinnitus loudness reduction after acoustic stimulation. On a group level all of the used stimuli induced short-term tinnitus suppression. Contrary to our hypothesis IBS appeared to produce the fewest reduction in tinnitus loudness rating, whereas IBP resulted in the strongest suppression pattern.

A potential explanation for this difference might derive from the ability of IBP/ WN in stimulating a broader range of frequencies around the ITF leading to a reduction of neural response gain and tinnitus-related hyperactivity and as a result facilitating shortterm tinnitus suppression (cf. Schaette et al. (2010)), whereas sup- 
A

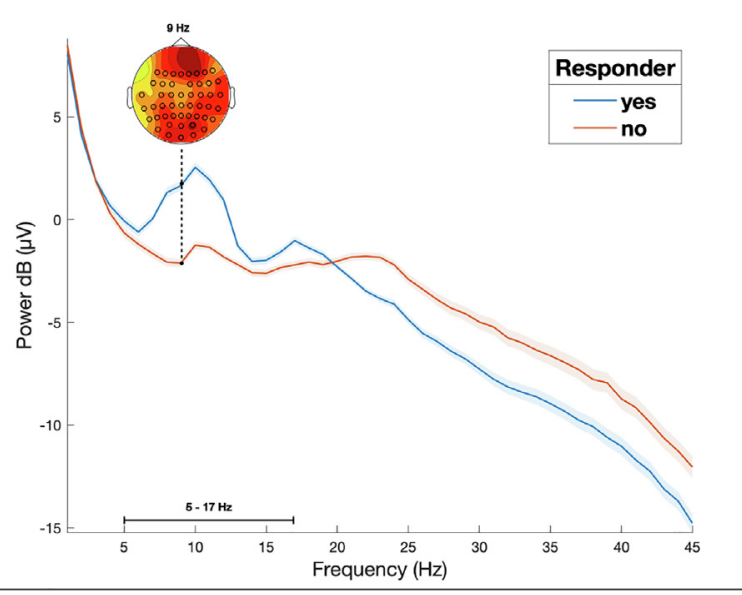

C

$9 \mathrm{~Hz}$ - right superior temporal gyrus

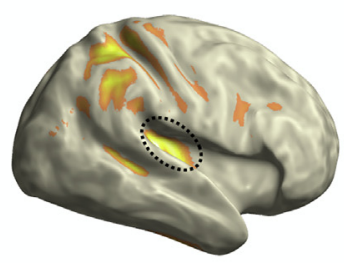

MNI [40 - 30 10]
B
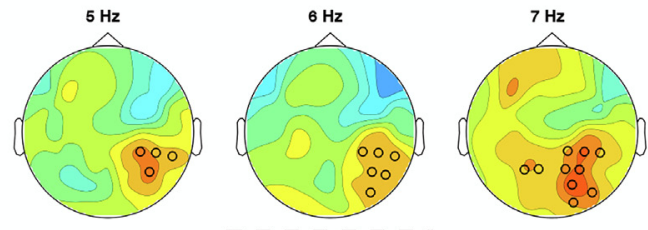

$\mathrm{Hz}$

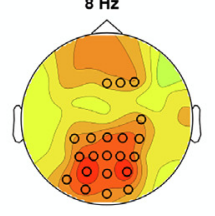

1 $-\overline{9}-\overline{H z}--1$
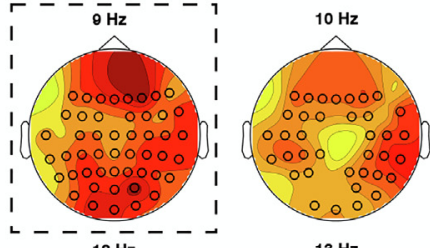

$13 \mathrm{~Hz}$
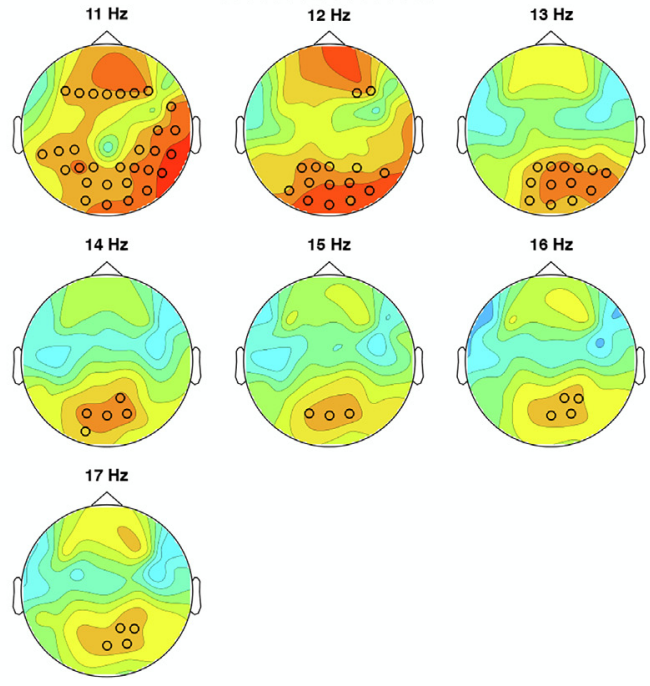

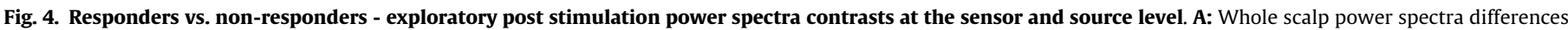

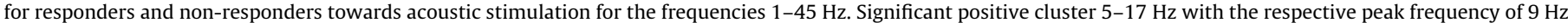

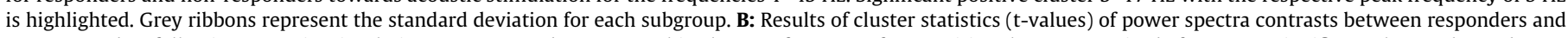

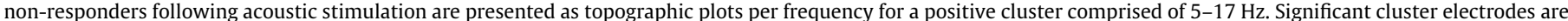

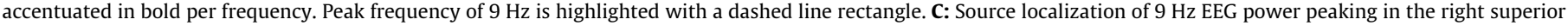
temporal gyrus (BA 41).

pressing effects of IBS via lateral inhibition might only appear after long-term application.

However, it is also possible that so called feed-forward inhibition is responsible for the superiority of stimuli containing signal in frequency ranges affected by hearing loss (cf. Roberts (2007, 2010)).

These explanations remain highly speculative and currently we are not able to provide a suitable explanation for these observed differences. Interestingly, stimulus IBP was evaluated with the lowest tolerability as indicated by the highest arousal and lowest valence ratings. This finding is contrary to one of our previous experiments which reports low arousal and high valence ratings for IBP (Schoisswohl et al., 2019).

Generally, about 50 to $90 \%$ of the studied individuals report some level of tinnitus suppression after acoustic stimulation (e.g., (Neff et al., 2017; Schoisswohl et al., 2019; Fournier et al., 2018; Kahlbrock and Weisz, 2008; Sedley et al., 2012)). Given the skewed distribution of RI responses on the group level in previous and this study as well as the need for a reliable threshold for strong tinnitus suppression, we opted to define a reduction in tinnitus of $50 \%$ after acoustic stimulation as the threshold for the responder classification akin to (Kahlbrock and Weisz, 2008). Applying this threshold, we can report an absolute number of 12 responders (with any stimulus type) out of 45 participants (26.67\% responder rate) which is comparable to relative numbers reported by Kahlbrock and Weisz (2008) (26\% responder rate), but below the quantity of responders reported by King et al. (2021) (56.67\% responder rate; the threshold for RI in this study is currently unknown due to publication status).

\subsection{Electrophysiology}

Since only a handful of studies evaluated neural activity during RI, no specific hypotheses were generated about oscillatory changes from pre to post stimulation. In light of past neurophysiological research and the assumptions that tinnitus is accompanied by abnormal delta, alpha and gamma activity (Weisz et al., 2005; Weisz et al., 2007; Adjamian et al., 2012; Moazami-Goudarzi et al., 2010; Balkenhol et al., 2013; van der Loo et al., 2009; Ashton et al., 2007) as well as a putative brief inversion of altered spontaneous brain activity during RI (Kahlbrock and Weisz, 2008), it can be supposed that observed group-level changes in tinnitus loudness (RI) are also reflected in electrophysiological measures. Namely, a reduction in delta and gamma and an increase in alpha power spectra from pre to post stimulation is to be expected given these assumptions.

\subsection{Whole group analysis}

Analysis of whole group pre-post stimulation changes in ongoing brain activity revealed increases in the delta, theta and gamma frequency range as well as decreases in alpha and beta 
frequency bands. This increase in low frequency activity is in direct contrast to past observations, which report a reduction of delta and theta power spectra during RI in accordance with the current neurophysiological models for tinnitus (Kahlbrock and Weisz, 2008; Sedley et al., 2012; Sedley et al., 2015). In contrast, an earlier study using neuromagnetic measures in a single subject during short-term tinnitus suppression likewise reports an enhancement of low frequency activity (Kristeva-Feige et al., 1995).

Gamma band activity was suggested to represent a spontaneous brain activity pattern related to the actual tinnitus perception (Weisz et al., 2007), therefore it is assumed that during a potential suppression of tinnitus after acoustic stimulation, activity in the gamma band will be suppressed. The current findings revealed an increase in gamma power after auditory stimulation, similar to findings from (King et al., 2021; Sedley et al., 2012, 2015), who observed an increase in gamma band activity during RI. Consistent with the current literature, we observed a decrease in alpha frequency band power from pre to post stimulation (Kahlbrock and Weisz, 2008; Sedley et al., 2015). However, a recent study was able to demonstrate an increase in alpha frequency band power during RI in accordance with the given neurophysiological models in tinnitus (King et al., 2021).

No relationship of pre-post power spectra changes, neither with tinnitus loudness ratings averaged over all time points nor directly after stimulation offset was observed in our data. Past neurophysiological research was not able to produce consistent findings in terms of correlations with behavioral measures of tinnitus respectively RI (e.g., intensity, loudness). Besides observed positive correlations of low and high frequency activity (Sedley et al., 2012; Balkenhol et al., 2013; van der Loo et al., 2009) or alpha activity with tinnitus intensity (Sedley et al., 2015; Meyer et al., 2014), the current findings are in accordance with other studies which report an absence of any relationship (Adjamian et al., 2012; Pierzycki et al., 2016; Kahlbrock and Weisz, 2008). In consideration of missing correlations as well as power spectra changes in conflict with current neurophysiological models for tinnitus, we suggest that the present findings do not indicate oscillatory patterns related to tinnitus loudness suppression, rather constitute a tinnitus-unspecific neurophysiological reaction to an external acoustic stimulus.

Oscillatory activity in the alpha frequency range is supposed to be relevant for inhibitory processes of the brain (Klimesch et al., 2007), thus a sound stimulation exceeding the individual tinnitus loudness level produces excitation and consequently alpha decreases. It has already been shown, that spontaneous activity in the alpha $(6-12 \mathrm{HZ})$ and beta $(20 \mathrm{~Hz})$ frequency bands desynchronize after sound stimulation (for an overview see Weisz et al. (2011)). Likewise, gamma band activity (30$45 \mathrm{~Hz} ; 80-100 \mathrm{~Hz}$ ), which is associated with cortical activation like attention or perception, was observed to be enhanced after the presentation of sound stimuli (Crone et al., 2001; Joliot et al., 1994) comparable to the present and recent findings (King et al., 2021).

In order to distinguish spontaneous brain activity related to tinnitus suppression from tinnitus-unspecific neurophysiological consequences to a sound stimulation, future research should not only compare acoustic stimulation responders and non-responders (RI vs. absence of RI) but also strive for a comparison with healthy control groups.

\subsection{Responder analysis}

Another objective of this study was to compare acoustic stimulation responders with non-responders, in order to point out potential differences in regards to ongoing brain activity. To the best of our knowledge this is the first study, which compares oscillatory activity of acoustic stimulation responders and nonresponders.

Interestingly, we observed reduced gamma band activity and a trend for enhanced alpha activity (peak frequency of $9 \mathrm{~Hz}$ localized in the right inferior temporal gyrus; BA 20) for the group of responders in contrast to non-responders. This result may corroborate the premise that gamma might be related to tinnitus perception (van der Loo et al., 2009; De Ridder et al., 2015; Ashton et al., 2007; Weisz et al., 2007). Given the fact, that responders generally reported their perceived tinnitus loudness level lower than non-responders, the question arises if the perceived tinnitus loudness rated via VAS can be associated with ongoing brain activity e.g., lower tinnitus loudness related to reduced gamma power or enhanced alpha. Yet, a respective correlation analysis failed to show an association.

As already shown by Schlee et al. (2014) tinnitus sufferers exhibited a blunted alpha peak and more importantly reduced alpha variability $(8-10 \mathrm{~Hz})$. This finding could be reflected by our data in a similar way as non-responders had a lower alpha peak and lower alpha variability $(8-12 \mathrm{~Hz})$. In further support for this argumentation, the data of the former study as well as our present findings show longer tinnitus duration for subjects with reduced alpha power, whereas we assume that these insights from casecontrol contrasts can be applied to the responder analysis at hand.

The observed reduction in gamma power may be interpreted along similar veins as the findings in alpha power by applying insights from case-control studies. Responders with a less chronified and intense tinnitus in our study are thus comparable to healthy controls in some case-control designs with reported lower gamma power values (Ashton et al., 2007; Vanneste et al., 2011). In further analogy, our findings of diminished gamma band activity together with a decrease in tinnitus loudness for the subgroup of responders can be linked to observations of past studies, namely a positive correlation of gamma with tinnitus loudness (van der Loo et al., 2009; De Ridder et al., 2015; Balkenhol et al., 2013).

We theorize that this trend for blunted alpha as well as lower gamma activity may be indicative of a trait as a consequence of tinnitus chronification.

A related observation was made by Neff et al. (2019) where active listening to tinnitus and consequential increase in tinnitus intensity did not lead to any neural alterations, which fits the reasoning about a trait-like neural representation of chronified tinnitus.

However, it is also possible that this pattern of reduced gamma and enhanced alpha activity represent a genuine neural trait related to acoustic stimulation response more specifically the possibility to induce RI in tinnitus sufferers.

Our exploratory analysis of post acoustic stimulation contrasts revealed higher spectral power in the theta, alpha and beta frequency range with a peak in the alpha band $(9 \mathrm{~Hz})$ localized in the right superior temporal gyrus (BA 41) in acoustic stimulation responders.

This increased alpha in auditory fields is in line with our hypothesis of a brief inversion of altered oscillatory power during $\mathrm{RI}$ and is consistent with past research examining disparities between tinnitus and healthy controls (compare Section 1). Notably, this supports our assumptions about responders and related trait-like neural signatures of tinnitus in that it surmises that only responders can exhibit neural responses which are specific to RI induced by acoustic stimulation.

Finally, a lack of correlations between loudness ratings and ongoing brain activity in the present study does not allow for a conclusive interpretation with regards to tinnitus. Past studies examining correlates of tinnitus suppression and neural activity have been able to demonstrate a relationship of low and high frequency activity with tinnitus intensity (Sedley et al., 2012, 2015). 
Nevertheless Kahlbrock and Weisz (2008) were not able to demonstrate a correlation of tinnitus suppression and ongoing neural activity in agreement with the present findings.

To further investigate these observed differences it is recommended to optimize future study designs with respect to a parametric analysis of tinnitus duration and RI-related neural activity.

\subsection{Limitations}

Our study has several limitations which might be informative for future research in the specific subfield of acoustic stimulation and general research in tinnitus.

The use of a standard boundary element headmodel as well as electrode positions hampers the accuracy of the source-level data in this study. Unfortunately, we could neither acquire individual structural MRIs nor register individual electrode positions for any of our participants.

No correlations between neurophysiological changes and changes in behaviorally assessed self-report tinnitus loudness were found in our data. Given the narrow and skewed distribution of the behavioral data and the consequential arbitrary choice of a RI threshold of $50 \%$ for the responder group contrast, correlation analysis might neither way be informative with the current data. This negative result is in line with the former study of Kahlbrock and Weisz (2008). Moreover, full and prolonged RI could only be studied in a small subset of the participants. Finally, heterogeneity of tinnitus loudness suppression curves between participants and the general low reliability and validity of tinnitus self-report data may further contribute to these absent findings.

As in many previous studies, it is challenging to recruit a large enough study sample from the locally available tinnitus population for the extensive experimental procedures. Additionally, tinnitus suppression responses, especially the parameters of RI depth as well as duration, can not be properly assessed in established screening procedures. This selection bias is hard to come by and potentially distorts results. Future studies could thus profit from internet-based or on-site pre-screenings in regards to the ability to (fully) suppress participants tinnitus acoustically (i.e., induce $\mathrm{RI}$ ) in order to generate a larger sample of responders, facilitating valid statements about oscillatory markers of RI. Beyond that, multi-center studies could help to further increase the validity of results aside from increasing the sample size.

\section{Conclusions}

The main goal of the current study was to unveil the oscillatory signature of RI and see how this relates to established neurophysiological models of tinnitus. In contrast to former studies, we used an extended set of modified noise stimuli targeting putatively differential neural mechanisms (i.e., RI and lateral inhibition). Furthermore, we explicitly investigated responder profiles of RI. Similar to former studies, merely a quarter of tested participants exhibited pronounced RI.

Looking at the oscillatory signature of acoustic stimulation responders and non-responders, results are indicative of decreased gamma and increased alpha power for responders. These findings are in line with both the proposed models of SLIM and TCD, respectively. This observations might be indicative of trait-specific forms of oscillatory signatures in different subsets of the tinnitus population possibly related to acoustic tinnitus suppression. In agreement with a potential transient reversal of tinnitus-specific abnormal ongoing brain activity over the course of tinnitus suppression, alpha power was enhanced in the group of responders after stimulation similarly compared to non-responders. Source localization of the sensor-level differences emphasizes the involvement of auditory cortical systems. Given the lack of correlations between tinnitus loudness and oscillatory power in this study, which was also reported by former studies, results do not allow for a conclusive interpretation with respect to these models.

The identified tinnitus patient profile experiencing RI, which mainly features less tinnitus chronification, could serve as a selection criterion to identify individuals for successful acoustic tinnitus suppression and putatively for acoustic treatments (e.g., treatment start in early stages of chronification).

Further research examining oscillatory activity during RI should strive for a healthy control group as well as control sounds not inducing RI in order to separate the neural signature of tinnitus suppression from tinnitus-unspecific neurophysiological effects.

\section{Funding}

This study was conducted as part of the European School for Interdisciplinary Tinnitus Research (ESIT (Schlee et al., 2018)). SS received funding from the European Union's Horizon 2020 research and innovation program under the Marie SklodowskaCurie grant (agreement number 722046). We furthermore thank the Swiss National Fund 'Early Postdoc Mobility' Grant P2ZHP1 174967 and to the University Research Priority Program 'Dynamics of Healthy Aging' of the University of Zurich for supporting PN during the preparation of the manuscript.

\section{Declaration of Competing Interest}

The authors declare that they have no known competing financial interests or personal relationships that could have appeared to influence the work reported in this paper.

\section{Acknowledgments}

We are thankful to all participants for their time and patience and especially grateful to Susanne Staudinger for her help with the study management. Moreover, we thank Anita Hafner and Bernhard Unsin for their valuable support in data acquisition and study management.

\section{Appendix A. Supplementary data}

Supplementary data associated with this article can be found, in the online version, at https://doi.org/10.1016/j.clinph.2021.03.038.

\section{References}

Adjamian P, Sereda M, Hall DA. The mechanisms of tinnitus: Perspectives from human functional neuroimaging. Hear Res 2009;253(1):15-31.

Adjamian P, Sereda M, Zobay O, Hall DA, Palmer AR. Neuromagnetic Indicators of Tinnitus and Tinnitus Masking in Patients with and without Hearing Loss. J Assoc Res Otolaryngol 2012;13(5):715-31.

Ashton H, Reid K, Marsh R, Johnson I, Alter K, Griffiths T. High frequency localised "hot spots" in temporal lobes of patients with intractable tinnitus: A quantitative electroencephalographic (QEEG) study. Neurosci Lett 2007;426 (1):23-8.

Balkenhol T, Wallhäusser-Franke E, Delb W. Psychoacoustic Tinnitus Loudness and Tinnitus-Related Distress Show Different Associations with Oscillatory Brain Activity. PLoS One 2013;8(1):e53180.

Basile C, Fournier P, Hutchins S, Hébert S. Psychoacoustic Assessment to Improve Tinnitus Diagnosis. PLoS One 2013;8(12):e82995.

Blaesing L, Goebel G, Floetzinger U, Berthold A, Kroener-Herwig B. Hypersensitivity to sound in tinnitus patients: an analysis of a construct based on questionnaire and audiological data. Int J Audiol 2010;49(7):518-26.

Bradley MM, Lang PJ. Measuring emotion: The self-assessment manikin and the semantic differential. J Behav Ther Exp Psychiatry 1994;25(1):49-59.

Brainard DH. The Psychophysics Toolbox. Spat Vis 1997;10(4):433-6.

Cederroth CR, Gallus S, Hall DA, Kleinjung T, Langguth B, Maruotti A, Meyer M, Norena A, Probst T, Pryss R, Searchfield G, Shekhawat G, Spiliopoulou M, Vanneste S, Schlee W. Editorial: Towards an Understanding of Tinnitus Heterogeneity. Front Aging Neurosci 2019;11:53. 
Cima RFF, Maes IH, Joore MA, Scheyen DJWM, El Refaie A, Baguley DM, Anteunis LJC, van Breukelen GJP, Vlaeyen JWS. Specialised treatment based on cognitive behaviour therapy versus usual care for tinnitus: a randomised controlled trial. Lancet 2012;379(9830):1951-9.

Croenlein T, Langguth B, Pregler M, Kreuzer PM, Wetter TC, Schecklmann M. Insomnia in patients with chronic tinnitus: Cognitive and emotional distress as moderator variables. J Psychosom Res 2016;83:65-8.

Crone, N.E., Boatman, D., Gordon, B., and Hao, L. (2001). Induced electrocorticographic gamma activity during auditory perception. Brazier Award-winning article, 2001. Clin. Neurophysiol, 112(4), 565-582.

De Ridder D, Congedo M, Vanneste S. The neural correlates of subjectively perceived and passively matched loudness perception in auditory phantom perception. Brain Behav 2015;5(5).

De Ridder D, Vanneste S, Langguth B, Llinas R. Thalamocortical Dysrhythmia: A Theoretical Update in Tinnitus. Front Neurol 2015;6.

Eggermont JJ. Pathophysiology of tinnitus. Prog. Brain Res. 2007;166:19-35.

Eggermont JJ, Roberts LE. The Neuroscience of Tinnitus: Understanding Abnormal and Normal Auditory Perception. Front Syst Neurosci 2012;6.

Eggermont JJ, Tass PA. Maladaptive Neural Synchrony in Tinnitus: Origin and Restoration. Front Neurol 2015;6.

Erlandsson S, Dauman N. Categorization of tinnitus in view of history and medical discourse. Int J Qual Stud Health Well-being 2013;8(1):23530.

Feldmann H. Homolateral and contralateral masking of tinnitus by noise-bands and by pure tones. Audiology 1971;10(3):138-44.

Feldmann H. Time Patterns and Related Parameters in Masking of Tinnitus. Acta Otolaryngol. 1983;95(5-6):594-8.

Fournier P, Cuvillier A-F, Gallego S, Paolino F, Paolino M, Quemar A, Londero A, Norena A. A New Method for Assessing Masking and Residual Inhibition of Tinnitus. Trends Hear 2018;22.

Fuchs M, Kastner J, Wagner M, Hawes S, Ebersole JS. A standardized boundary element method volume conductor model. Clin. Neurophysiol. 2002;113 (5):702-12.

Fuller T, Cima R, Langguth B, Mazurek B, Vlaeyen JW, Hoare DJ. Cognitive behavioural therapy for tinnitus. Cochrane Database Syst Rev 2020;1(1): CD012614.

Galazyuk AV, Longenecker RJ, Voytenko SV, Kristaponyte I, Nelson GL. Residual inhibition: From the putative mechanisms to potential tinnitus treatment. Hear Res 2019;375:1-13.

Galazyuk AV, Voytenko SV, Longenecker RJ. Long-Lasting forward Suppression of Spontaneous Firing in Auditory Neurons: Implication to the Residual Inhibition of Tinnitus. J Assoc Res Otolaryngol 2017;18(2):343-53.

Goebel G, Hiller W. The tinnitus questionnaire. A standard instrument for grading the degree of tinnitus. Results of a multicenter study with the tinnitus questionnaire. HNO 1994;42(3):166-72.

Green, D.M. (1993). A maximum-likelihood method for estimating thresholds in a yes-no task. J Acoust Soc Am, 93(4, Pt 1):2096-2105.

Groß J, Kujala J, Hämäläinen M, Timmermann L, Schnitzler A, Salmelin R. Dynamic imaging of coherent sources: studying neural interactions in the human brain. Proc. Nat. Acad. Sci. 2001;98(2):694-9.

Hallam RS, Jakes SC, Hinchcliffe R. Cognitive variables in tinnitus annoyance. Br J Clin Psychol 1988;27(Pt 3):213-22.

Harrison XA, Donaldson L, Correa-Cano ME, Evans J, Fisher DN, Goodwin CE, Robinson BS, Hodgson DJ, Inger R. A brief introduction to mixed effects modelling and multi-model inference in ecology. PeerJ 2018;6:e4794.

Heller AJ. Classification and epidemiology of tinnitus. Otolaryngol Clin North Am 2003;36(2):239-48.

Henry JA, Roberts LE, Ellingson RM, Thielman EJ. Computer-Automated Tinnitus Assessment: Noise-Band Matching, Maskability, and Residual Inhibition. J Am Acad Audiol 2013;24(6):486-504.

Hesse G. Evidence and evidence gaps in tinnitus therapy. GMS Curr Top Otorhinolaryngol. Head Neck Surg 2016;15(Doc04).

Joliot M, Ribary U, Llinás R. Human oscillatory brain activity near $40 \mathrm{~Hz}$ coexists with cognitive temporal binding. PNAS USA 1994;91(24):11748-51.

Kahlbrock N, Weisz N. Transient reduction of tinnitus intensity is marked by concomitant reductions of delta band power. BMC Biol 2008;6(1):4.

King ROC, Singh Shekhawat G, King C, Chan E, Kobayashi K, Searchfield GD. The Effect of Auditory Residual Inhibition on Tinnitus and the Electroencephalogram. Ear Hear. 2021;42(1):130-41.

Kleiner M, Brainard D, Pelli D, Ingling A, Murray R, Broussard C. What's new in psychtoolbox-3. Perception 2007;36(14):1-16.

Kleinjung, T., Fischer, B., Langguth, B., Sand, P.G., Hajak, G., Dvorakova, J., and Eichhammer, P. (2007). Validierung einer deutschsprachigen Version des Tinnitus Handicap Inventory. Psychiat Prax, 34(S 1):S140-S142.

Kleinjung T, Langguth B. Avenue for Future Tinnitus Treatments. Otolaryngol Clin North Am 2020:53(4):667-83.

Klimesch W, Sauseng P, Hanslmayr S. EEG alpha oscillations: the inhibition-timing hypothesis. Brain Res Rev 2007;53(1):63-88.

Kristeva-Feige R, Feige B, Kowalik Z, Ross B. Neuromagnetic activity during residual inhibition in tinnitus. J Audiol Med 1995;4(3):135-42.

Landry EC, Sandoval XCR, Simeone CN, Tidball G, Lea J, Westerberg BD. Systematic Review and Network Meta-analysis of Cognitive and/or Behavioral Therapies (CBT) for Tinnitus. Otol Neurotol 2020;41(2):153-66.

Langguth B, Goodey R, Azevedo A, Bjorne A, Cacace A, Crocetti A, Del Bo L, De Ridder D, Diges I, Elbert T, Flor H, Herraiz C, Ganz Sanchez T, Eichhammer P, Figueiredo R, Hajak G, Kleinjung T, Landgrebe M, Londero A, Lainez MJA, Mazzoli M, Meikle MB, Melcher J, Rauschecker JP, Sand PG, Struve M, Van de Heyning P, Van Dijk P,
Vergara R. Consensus for tinnitus patient assessment and treatment outcome measurement: Tinnitus Research Initiative meeting, Regensburg, July 2006. Prog. Brain Res. 2007;166:525-36.

Langguth B, Kreuzer PM, Kleinjung T, De Ridder D. Tinnitus: causes and clinical management. Lancet Neurol 2013;12(9):920-30.

Leiner, D.J. (2016). Sosci survey (version 3.0.01-i). retrieved from https:// www.soscisurvey.de.

Li J, Jin J, Xi S, Zhu Q, Chen Y, Huang M, He C. Clinical efficacy of cognitive behavioral therapy for chronic subjective tinnitus. Am J Otolaryngol 2019;40(2):253-6.

Llinás R, Urbano FJ, Leznik E, Ramírez RR, van Marle HJF. Rhythmic and dysrhythmic thalamocortical dynamics: GABA systems and the edge effect. Trends Neurosci 2005;28(6):325-33.

Llinás RR, Ribary U, Jeanmonod D, Kronberg E, Mitra PP. Thalamocortical dysrhythmia: A neurological and neuropsychiatric syndrome characterized by magnetoencephalography. PNAS USA 1999;96(26):15222-7.

Mazurek B, Olze H, Haupt H, Szczepek AJ. The More the Worse: the Grade of NoiseInduced Hearing Loss Associates with the Severity of Tinnitus. Int. J. Environ. Res. Public Health 2010;7(8):3071-9.

Meyer, M., Luethi, M.S., Neff, P., Langer, N., and Büchi, S. (2014). Disentangling Tinnitus Distress and Tinnitus Presence by Means of EEG Power Analysis. Neural Plast, 2014.

Moazami-Goudarzi M, Michels L, Weisz N, Jeanmonod D. Temporo-insular enhancement of EEG low and high frequencies in patients with chronic tinnitus. QEEG study of chronic tinnitus patients. BMC Neurosci 2010;11.

Nakagawa S, Johnson PCD, Schielzeth H. The coefficient of determination R2 and intra-class correlation coefficient from generalized linear mixed-effects models revisited and expanded. J R Soc Interface 2017;14(134).

Neff P, Hemsley C, Kraxner F, Weidt S, Kleinjung T, Meyer M. Active listening to tinnitus and its relation to resting state EEG activity. Neurosci Lett 2019;694:176-83.

Neff, P., Michels, J., Meyer, M., Schecklmann, M., Langguth, B., and Schlee, W. (2017). $10 \mathrm{~Hz}$ Amplitude Modulated Sounds Induce Short-Term Tinnitus Suppression. Front Aging Neurosci, 9.

Neff P, Zielonka L, Meyer M, Langguth B, Schecklmann M, Schlee W. Comparison of Amplitude Modulated Sounds and Pure Tones at the Tinnitus Frequency: Residual Tinnitus Suppression and Stimulus Evaluation. Trends Hear 2019;23. 2331216519833841.

Newman CW, Wharton JA, Shivapuja BG, Jacobson GP. Relationships among Psychoacoustic Judgments, Speech Understanding Ability and Self-Perceived Handicap in Tinnitus Subjects. Int J Audiol 1994;33(1):47-60.

Nondahl DM, Cruickshanks KJ, Dalton DS, Klein BEK, Klein R, Schubert CR, Tweed TS Wiley TL. The impact of tinnitus on quality of life in older adults. J Am Acad Audiol 2007; 18(3):257-66.

Norena A, Micheyl C, Chéry-Croze S, Collet L. Psychoacoustic Characterization of the Tinnitus Spectrum: Implications for the Underlying Mechanisms of Tinnitus. Audiol Neurotol 2002;7(6):358-69.

Okamoto H, Stracke H, Stoll W, Pantev C. Listening to tailor-made notched music reduces tinnitus loudness and tinnitus-related auditory cortex activity. PNAS 2010;107(3):1207-10.

Oostenveld R, Fries P, Maris E, Schoffelen J-M. FieldTrip: Open Source Software for Advanced Analysis of MEG, EEG, and Invasive Electrophysiological Data. Comput Intell Neurosci 2011;2011:1-9.

Oostenveld R, Stegeman DF, Praamstra P, van Oosterom A. Brain symmetry and topographic analysis of lateralized event-related potentials. Clinical neurophysiology 2003;114(7):1194-202.

Oostenveld R, Stegeman DF, Praamstra P, van Oosterom A. Brain symmetry and topographic analysis of lateralized event-related potentials. Clin. Neurophysiol. 2003;114(7):1194-202.

Pantev C, Okamoto H, Teismann H. Music-induced cortical plasticity and lateral inhibition in the human auditory cortex as foundations for tonal tinnitus treatment. Front Syst Neurosci 2012;6.

Pierzycki RH, McNamara AJ, Hoare DJ, Hall DA. Whole scalp resting state EEG of oscillatory brain activity shows no parametric relationship with psychoacoustic and psychosocial assessment of tinnitus: A repeated measures study. Hear Res 2016;331:101-8.

Reavis KM, Rothholtz VS, Tang Q, Carroll JA, Djalilian H, Zeng F-G. Temporary Suppression of Tinnitus by Modulated Sounds. J Assoc Res Otolaryngol 2012;13 (4):561-71.

Roberts LE. Residual inhibition. Prog Brain Res 2007;166:487-95.

Roberts LE, Eggermont JJ, Caspary DM, Shore SE, Melcher JR, Kaltenbach JA. Ringing Ears: The Neuroscience of Tinnitus. J Neurosci 2010;30(45):14972-9.

Roberts LE, Moffat G, Baumann M, Ward LM, Bosnyak DJ. Residual Inhibition Functions Overlap Tinnitus Spectra and the Region of Auditory Threshold Shift. J Assoc Res Otolaryngol 2008;9(4):417-35.

Roberts LE, Moffat G, Bosnyak DJ. Residual inhibition functions in relation to tinnitus spectra and auditory threshold shift. Acta Otolaryngol. 2006;126 (sup556):27-33.

Schaette R, Koenig O, Hornig D, Gross M, Kempter R. Acoustic stimulation treatments against tinnitus could be most effective when tinnitus pitch is within the stimulated frequency range. Hear Res 2010;269(1-2):95-101.

Schecklmann M, Vielsmeier V, Steffens T, Landgrebe M, Langguth B, Kleinjung T. Relationship between Audiometric Slope and Tinnitus Pitch in Tinnitus Patients: Insights into the Mechanisms of Tinnitus Generation. PLoS One 2012;7(4).

Schlee W, Hall DA, Canlon B, Cima RFF, de Kleine E, Hauck F, Huber A, Gallus S, Kleinjung T, Kypraios T, Langguth B, Lopez-Escamez JA, Lugo A, Meyer M, 
Mielczarek P, Norena A, Pfiffner F, Pryss RC, Reichert M, Requena T, Schecklmann M, van Dijk P, van de Heyning P, Weisz N, Cederroth CR. Innovations in Doctoral Training and Research on Tinnitus: The European School on Interdisciplinary Tinnitus Research (ESIT) Perspective. Front Aging Neurosci 2018:9.

Schlee W, Schecklmann M, Lehner A, Kreuzer PM, Vielsmeier V, Poeppl TB, Langguth B. Reduced Variability of Auditory Alpha Activity in Chronic Tinnitus. Neural Plast 2014;2014:1-9.

Schoisswohl S, Arnds J, Schecklmann M, Langguth B, Schlee W, Neff P. Amplitude Modulated Noise for Tinnitus Suppression in Tonal and Noise-Like Tinnitus. Audiol Neurotol 2019;24(6):309-21.

Sedley W, Gander P, Kumar S, Oya H, Kovach C, Nourski K, Kawasaki H, Howard M, Griffiths T. Intracranial Mapping of a Cortical Tinnitus System using Residual Inhibition. Curr Biol 2015;25(9):1208-14.

Sedley W, Teki S, Kumar S, Barnes GR, Bamiou D-E, Griffiths TD. Single-subject oscillatory gamma responses in tinnitus. Brain 2012;135(10):3089-100.

Trevis KJ, McLachlan NM, Wilson SJ. Psychological mediators of chronic tinnitus: The critical role of depression. J Affect Disord 2016;204:234-40.

Tyler R, Stocking C, Secor C, Slattery WH. Amplitude Modulated S-Tones Can Be Superior to Noise for Tinnitus Reduction. Am J Audiol 2014;23(3):303.

van der Loo E, Gais S, Congedo M, Vanneste S, Plazier M, Menovsky T, Van de Heyning P, De Ridder D. Tinnitus intensity dependent gamma oscillations of the contralateral auditory cortex. PLoS One 2009;4(10):e7396.
Vanneste S, Heyning PVd, Ridder DD. Contralateral parahippocampal gamma-band activity determines noise-like tinnitus laterality: a region of interest analysis. Neuroscience 2011;199:481-90.

Vernon JA, Meikle MB. Tinnitus: clinical measurement. Otolaryngol Clin North Am 2003:36(2):293-305.

Weidt S, Delsignore A, Meyer M, Rufer M, Peter N, Drabe N, Kleinjung T. Which tinnitus-related characteristics affect current health-related quality of life and depression? A cross-sectional cohort study. Psychiatry Res 2016;237:114-21.

Weisz N, Dohrmann K, Elbert T. The relevance of spontaneous activity for the coding of the tinnitus sensation. Prog Brain Res 2007;166:61-70.

Weisz N, Hartmann T, Müller N, Lorenz I, Obleser J. Alpha Rhythms in Audition. Cognitive and Clinical Perspectives. Front Psychol 2011;2.

Weisz N, Müller S, Schlee W, Dohrmann K, Hartmann T, Elbert T. The neural code of auditory phantom perception. J. Neurosci 2007;27(6):1479-84.

Weisz N, Moratti S, Meinzer M, Dohrmann K, Elbert T. Tinnitus Perception and Distress Is Related to Abnormal Spontaneous Brain Activity as Measured by Magnetoencephalography. PLoS Med 2005;2(6):e153.

Zenner H-P, Delb W, Kroener-Herwig B, Jaeger B, Peroz I, Hesse G, Mazurek B, Goebel G, Gerloff C, Trollmann R, Biesinger E, Seidler H, Langguth B. A multidisciplinary systematic review of the treatment for chronic idiopathic tinnitus. Eur Arch Otorhinolaryngol 2017;274(5):2079-91.

Zobay O, Palmer AR, Hall DA, Sereda M, Adjamian P. Source Space Estimation of Oscillatory Power and Brain Connectivity in Tinnitus. PLoS One 2015;10(3): e0120123. 